\title{
Seguimento de Regras Nutricionais em Crianças com Excesso de Peso
}

\author{
Lana Cristina Cardoso de Oliveira Martins ${ }^{1}$ \\ Eleonora Arnaud Pereira Ferreira \\ Lúcia Cristina Cavalcante da Silva \\ Flávia Pinho Almeida \\ Universidade Federal do Pará
}

\begin{abstract}
RESUMO - Foram avaliados efeitos de instruções, treino de relato verbal (TRV) e treino de automonitoração (TA), com e sem a presença do cuidador principal, sobre o seguimento de regras nutricionais em crianças com excesso de peso. Participaram duas crianças (P1: menino de 9 anos; P2: menina de 11 anos) e suas cuidadoras, por meio de entrevistas em ambulatório. Após instruções, P1 manteve e P2 melhorou o conhecimento das orientações nutricionais. Os Índices de adesão à dieta obtidos por P2 foram mais elevados do que os obtidos por P1 em todas as fases. Houve mudança com significância clínica após introdução de TRV em ambos os participantes. Discute-se a eficácia dos procedimentos de intervenção utilizados e a importância da presença do cuidador.
\end{abstract}

Palavras-chave: obesidade, sobrepeso, cuidadores, instruções, automonitoração, relato verbal

\section{Following Nutritional Rules in Overweight Children}

\begin{abstract}
This study assessed the effects of instructions, verbal report training (VRT) and self-monitoring training (ST), with and without the presence of the primary caregiver, on following nutritional rules in obese or overweight children. Two children (P1: a nine-year-old boy; P2: a eleven-year-old girl) and their caregivers participated through interviews performed in an outpatient clinic. Ten interviews were performed. After instructions, P1 maintained and P2 improved the knowledge on nutritional orientation. Adherence to diet scores obtained by P2 were higher than those obtained by P1 in all phases. There was a clinically significant change after the introduction of the VRT in both participants. Efficiency of the intervention procedures used and the importance of the caregiver's presence are discussed.
\end{abstract}

Keywords: obesity, overweight, caregiver, instructions, self-monitoring, verbal report

O excesso de peso em crianças é um tema de relevância social e tem sido investigado por diversas áreas do conhecimento, dentre estas, a Análise do Comportamento. Esta área considera que problemas de comportamento, como o comer em excesso, podem ser resolvidos a partir da construção e/ou ampliação de repertórios comportamentais, mais do que por meio da eliminação de repertórios (Goldiamond, 1974/2002; Skinner, 1969/1975).

O tratamento do excesso de peso - como a obesidade e o sobrepeso - requer a adesão dos pacientes a orientações de equipes de saúde relacionadas ao comportamento alimentar e à prática de atividade física. Malerbi (2000) sugere que uma baixa adesão é mais frequente quando o tratamento é longo, complexo, preventivo e quando requer mudanças no estilo de vida do paciente, como é o caso do excesso de peso. Além disso, a baixa adesão, segundo Malerbi (2000), deve-se ao fato de que as complicações decorrentes da doença provavelmente ocorrerão somente no futuro (consequências tardias) e, ainda, é muito importante que os profissionais considerem o repertório de entrada dos pacientes e que iniciem o tratamento com mudanças graduais no estilo de vida.

Adesão ao tratamento requer seguimento de instruções de profissionais da área da saúde, e estas podem ser consideradas como exemplos de regras. Segundo Albuquerque (2001),

1 Endereço para correspondência: Rua Augusto Correa, 01, Cidade Universitária, Campus do Guamá, Núcleo de Teoria e Pesquisa do Comportamento. Belém-PA, Brasil. CEP: 66075-110. E-mail: lanacardoso@hotmail.com, regras podem exercer múltiplas funções como, por exemplo, de estímulo discriminativo (Baum, 2006; Skinner, 1969/1975) e de estímulos alteradores de função de outros estímulos (Blakely \& Schlinger, 1987; Schlinger \& Blakely, 1987). Segundo Albuquerque e Ferreira (2001), a extensão da regra dificulta o seguimento da mesma. E ainda, de acordo com Albuquerque, Paracampo e Albuquerque (2004) a perda de reforçadores torna o seguimento de regras menos provável, mesmo quando monitorado, como é o caso do tratamento do excesso de peso.

O uso de técnicas comportamentais como automonitoração e reforçamento, utilizadas tanto em atendimentos individuais quanto em grupo, mostram-se eficazes na adesão ao tratamento do excesso de peso em crianças (Epstein et al., 1985; Fisberg, 2004; Gunnarsdottir, Sigurdardottir, Njardvik, Olafsdottir, \& Bjarnason, 2011; Robertson, Thorogood, Inglis, Grainger, \& Stewart-Brown, 2011). Entretanto, segundo Hidaka (2007), é preciso cuidado com as omissões nos registros de automonitoração feitos por crianças, além da necessidade de se adequar os questionários a fim de facilitar a compreensão pelas crianças.

Além de técnicas comportamentais, tem-se destacado a importância da associação entre excesso de peso e conhecimento sobre a doença e suas consequências. No estudo de Triches e Giugliani (2005), que investigou a relação entre obesidade e práticas alimentares e conhecimento sobre nutrição em crianças, os resultados demonstraram que práticas alimentares saudáveis estavam associadas a um maior conhecimento em nutrição, uma vez que crianças com 
menos conhecimento e práticas alimentares menos saudáveis tinham cinco vezes mais chance de serem obesas. Por sua vez, outros estudos consideram que ter conhecimento não implica no seguimento das orientações nutricionais, como observado por Fisberg (2004) e por Kopelman, Roberts e Adab (2007).

Além do fornecimento de instruções, pesquisas utilizaram treinos de relato verbal e de automonitoração para promover adesão ao tratamento tanto em adultos (Ferreira \& Fernandes, 2009; Immer, 2005; Nobre, 2010) quanto em crianças (Bentes, 2011; Hidaka, 2007; Soares \& Costa, 2011) com excesso de peso. Os resultados sugerem que o uso de tais treinos promoveu aumento na ocorrência de respostas de auto-observação do comportamento alimentar e nos índices de adesão à dieta na amostra estudada.

Em se tratando de crianças, os cuidadores são geralmente os responsáveis pela modelagem e reforçamento do comportamento alimentar, assim como pelo contexto em que este ocorre. Em geral, cabe ao cuidador a compra e o preparo do alimento que será oferecido à criança, servindo como modelo de escolha alimentar saudável ou não (Fisher, Sinton, \& Birch, 2009; Lindsay, Sussner, Kim, \& Gortmaker, 2006; Rossi, Moreira, \& Rauen, 2008; Valverde \& Patin, 2004).

Estudos realizados com crianças com excesso de peso e seus cuidadores, como o de Germann, Kirschenbaum e Rich (2007) e o de Kirschenbaum, Harris e Tomarken (1984), verificaram que quando os cuidadores estavam envolvidos na condição experimental, os resultados eram mais favoráveis à redução de peso e/ou manutenção da perda de peso. Por sua vez, no estudo de Laessle, Uhl, Lindel e Müller (2001) foi manipulada a presença da mãe na sessão e os resultados apontaram que crianças obesas comiam mais rapidamente e em porções maiores quando estavam acompanhadas pelas mães. No estudo de Lent, Hill, Dollahite, Wolfe e Dickin (2012), realizado por meio de uma intervenção com crianças e pais na qual foram fornecidas informações sobre nutrição e atividade física além de intervenções comportamentais, os resultados indicaram que houve mudança no comportamento dos participantes, como redução no consumo de refrigerante e aumento no consumo de verduras e frutas, e ainda, que outros membros da família também foram beneficiados com o tratamento.

Observa-se que ainda não está claro quais os procedimentos mais eficazes no controle do excesso de peso em crianças, nem sobre a participação do cuidador neste processo.

O objetivo deste estudo foi avaliar os efeitos combinados de instruções, do treino de relato verbal e do treino de automonitoração, aplicados sem (Condição 1) e com (Condição 2) a presença do cuidador principal, sobre o seguimento de regras nutricionais em crianças com excesso de peso.

\section{Método}

\section{Participantes}

Participaram deste estudo duas crianças e suas cuidadoras primárias, selecionadas a partir dos seguintes critérios: (a) a criança deveria ter obesidade ou sobrepeso, com ausência de comorbidades, a partir de diagnóstico emitido por profissional de nutrição do ambulatório de um hospital universitário (HU); estar há, no mínimo, com seis meses de tratamento no ambulatório; ter ausência de história prévia de treinamento semelhante ao do estudo; apresentar dificuldade de adesão ao tratamento, identificada pelo encaminhamento do profissional de nutrição do HU; estar cursando a partir da $2^{\mathrm{a}}$ série do Ensino Fundamental, sugerindo ter habilidades de leitura e escrita; e, concordar em participar voluntariamente da pesquisa; (b) a cuidadora deveria ter idade igual ou superior a 18 anos e concordar em participar da pesquisa, por meio da assinatura do Termo de Consentimento Livre e Esclarecido [TCLE]. Os critérios de exclusão adotados foram a criança ter dificuldade ou transtorno de aprendizagem, ou ter diagnóstico de alguma síndrome genética.

Foram encaminhadas pelo profissional de nutrição do HU cinco crianças em acompanhamento ambulatorial, que preencheram os critérios de inclusão. Contudo, houve um caso de abandono e dois casos de desligamento decorrentes de constantes remarcações das entrevistas o que impossibilitaria a conclusão da coleta de dados em tempo hábil.

\section{Caracterização dos participantes}

Condição 1 (sem a presença da cuidadora):

Participante 1 (P1): sexo masculino, 9 anos de idade, $3^{\text {a }}$ série do Ensino Fundamental, com um ano e 11 meses de atendimento no Serviço de Nutrição do HU, diagnóstico de obesidade (IMC/I= >P97). Cuidador 1 (C1): mãe de P1, 29 anos de idade, dona de casa, com Ensino Médio completo, Classe C2 de acordo com o critério de classificação econômica Brasil da Associação Brasileira de Empresas de Pesquisa [ABEP] (2010).

Condição 2 (com a presença da cuidadora):

Participante 2 (P2): sexo feminino, 11 anos de idade, $5^{\mathrm{a}}$ série do Ensino Fundamental, acompanhada pelo Serviço de Nutrição do HU pelo período de um ano, diagnóstico de sobrepeso (IMC/I: P85-P97). Cuidador 2 (C2): mãe de P2, 52 anos de idade, auxiliar de serviços gerais, com Ensino Médio completo, Classe C2, segundo a ABEP (2010).

\section{Ambiente}

A coleta de dados foi realizada em um consultório do ambulatório de psicologia do HU, adaptado para o atendimento da clientela infantil.

\section{Instrumentos}

Roteiro de entrevista: adaptado de Nobre (2010), composto por questões relativas ao conhecimento dos participantes sobre obesidade, às orientações recebidas durante a última consulta com a nutricionista, sobre hábitos alimentares e prática regular de atividade física. 
Recordatório 24 horas: roteiro de entrevista para levantamento sobre horário das refeições, tipo e quantidade de alimentos ingeridos, descrição do ambiente físico e social (incluindo antecedentes e consequentes relacionados ao contexto das refeições). Foi aplicado tendo como referência o comportamento alimentar emitido nas 24 horas do dia anterior à entrevista, considerando cada refeição realizada.

Manual informativo sobre obesidade e alimentação saudável: produzido para este estudo sob a supervisão de uma nutricionista e a partir de publicações sobre o tema disponíveis na literatura da área. Ilustrado com instruções sobre obesidade - suas causas, consequências e tratamentos -, comportamentos que devem ser emitidos para a obtenção de um estilo de vida saudável e com as pirâmides alimentar e de atividade física. Foi elaborado com o objetivo de instruir os participantes sobre obesidade e alimentação saudável.

Protocolo de orientação nutricional para crianças: documento fornecido pelo profissional de nutrição do HU, contendo as orientações (regras nutricionais) indicadas para cada participante, bem como a lista de substituição de alimentos. Foi utilizado com o objetivo de analisar a concordância entre os relatos dos participantes acerca do comportamento alimentar e as orientações nutricionais, permitindo o cálculo do Índice de Adesão à Dieta (IAD) a partir da fórmula sugerida por Nobre (2010).

Formulário de automonitoração: adaptado do modelo proposto por Hidaka (2007), composto por figuras correspondentes a alimentos frequentemente ingeridos por crianças em cada uma de seis refeições diárias (desjejum, lanche da manhã, almoço, lanche da tarde, jantar e ceia). Neste formulário, as crianças são instruídas a marcar com um $\mathrm{X}$ os alimentos ingeridos em cada refeição ao longo do dia, podendo acrescentar o nome do alimento ingerido que não estivesse representado por figura.

Roteiro para análise do formulário de automonitoração: roteiro de entrevista com questões para investigar a concordância entre os registros feitos pela criança nos formulários de automonitoração e as regras contidas no protocolo de orientação nutricional, além de perguntas sobre o que a criança poderia fazer para melhorar a adesão ao tratamento.

\section{Procedimento de coleta de dados}

Foi realizado um estudo com delineamento longitudinal de curto prazo, com o sujeito como seu próprio controle. A coleta de dados ocorreu em 10 sessões distribuídas em aproximadamente 15 semanas, com intervalo mínimo de sete dias entre as sessões.

Após aprovação do projeto pelo Comitê de Ética em Pesquisa (CAAE 0495.0.000.073-11; Parecer $\mathrm{n}^{\circ}$ 062/11), a coleta de dados ocorreu de acordo com as seguintes etapas:

Inicialmente, foi realizado contato com o serviço de nutrição do HU para apresentação dos objetivos da pesquisa e orientação sobre o encaminhamento dos candidatos a participantes. Posteriormente, foi agendada a primeira sessão com o cuidador e com a criança, na qual foram confirmados os critérios de inclusão, explicados os objetivos do estudo e a assinatura do TCLE. Em seguida, os participantes foram inseridos, por ordem de entrada no estudo, em uma de duas condições. Na Condição 1, apenas a criança estava presente nas sessões de linha de base e de intervenção. $\mathrm{Na}$ Condição 2 , tanto a criança quanto seu cuidador primário estavam presentes nas sessões de linha de base e de intervenção. Em ambas as condições, o foco da intervenção era a criança, sendo a participação do cuidador na Condição 2 restrita a ouvir as orientações da pesquisadora ou prestar esclarecimentos quando houvesse necessidade.

Na segunda sessão, etapa de Linha de Base 1 (LB1), os participantes responderam inicialmente ao Roteiro de entrevista seguido da aplicação do Recordatório 24 horas. Em seguida, os participantes receberam instruções sobre obesidade e comportamento alimentar por meio do Manual informativo sobre obesidade e alimentação saudável, o qual era lido juntamente com a pesquisadora.

Prosseguindo, as crianças foram submetidas ao treino de relato verbal (TRV) realizado na terceira e na quarta sessão, com intervalo mínimo de sete dias, durante as quais foi aplicado o Recordatório 24 horas. Nestas entrevistas era feita análise de concordância entre o que o participante relatava sobre sua alimentação e as orientações nutricionais descritas no Protocolo de orientação nutricional para crianças. As descrições de comportamentos coincidentes com os comportamentos prescritos para o tratamento eram consequenciadas por meio de reforçamento positivo, na forma de elogios orais emitidos pela pesquisadora. Essas análises permaneceram durante todas as sessões de coleta de dados.

Na quinta sessão, etapa de Linha de Base 2 (LB2), inicialmente foi aplicado o Recordatório 24 horas e, em seguida, os participantes foram ensinados a preencher o modelo de Formulário de automonitoração, tendo como exemplo a alimentação realizada pela criança no dia anterior à entrevista. A partir de então, as crianças foram submetidas ao treino em registro de automonitoração (TA), nas sexta, sétima e oitava sessões, com intervalo mínimo de sete dias. Nas sessões anteriores (quinta, sexta e sétima), os participantes receberam Formulários de automonitoração para preenchimento em domicílio e deveriam apresentálos devidamente preenchidos nas sessões seguintes. No TA eram feitas análises dos registros preenchidos pelos participantes, seguindo o Roteiro para análise do formulário de automonitoração complementadas com a aplicação do Recordatório 24 horas.

Decorridas cinco semanas após a realização da terceira entrevista de TA (correspondente à oitava sessão), foi realizada a nona sessão (Follow-up 1-F1), com a aplicação do Roteiro de entrevista e do Recordatório 24 horas. Após duas semanas, fez-se uma entrevista de encerramento (décima sessão: Follow-up 2 - F2) na qual foi aplicado o Recordatório 24 horas. Ao final desta entrevista foi realizada uma devolutiva aos participantes acerca dos resultados obtidos.

Todas as sessões de coleta de dados foram gravadas em áudio e encerravam com atividades lúdicas com as crianças, utilizando-se jogos e brinquedos disponíveis na sala do ambulatório de psicologia do HU. O objetivo dessas 
atividades era tornar as sessões reforçadoras para a criança incentivando seu comparecimento às entrevistas da pesquisa.

\section{Procedimento de análise dos dados}

Os dados obtidos por meio do Roteiro de entrevista permitiram a elaboração de categorias de análise dos relatos dos participantes. A seleção destas categorias foi feita por meio de um banco de dados construído a partir das informações contidas no Manual informativo sobre obesidade e alimentação saudável e no Protocolo de orientação nutricional para crianças. Em seguida, estas categorias foram analisadas por dois juízes independentes, para a obtenção do cálculo de concordância, utilizando-se a fórmula: número de concordâncias dividido pela soma entre as concordâncias e as discordâncias, sendo o resultado multiplicado por 100 (Fagundes, 1982). Obteve-se um índice de 100\% de concordância entre os juízes independentes.

As categorias de análise utilizadas foram as seguintes:

(1) Conhecimentos sobre obesidade

Nesta categoria foram investigados os conhecimentos dos participantes sobre cinco itens: definição, causas, consequências imediatas, consequências em longo prazo e tratamento. No item Definição, os tópicos que serviam como referência para analisar a resposta do participante como correta ou não foram os seguintes: doença, excesso de gordura, prejuízo à saúde e cronicidade da doença. Quanto ao item Causas, os tópicos foram alimentação inadequada e sedentarismo. Quanto às Consequências imediatas, utilizouse como referência: dores nos joelhos, dificuldade para respirar quando faz esforço físico, perda de roupas mais rapidamente. Na análise das Consequências em longo prazo, os tópicos indicados foram doenças do coração, doenças respiratórias, doenças psicológicas, pressão alta, problemas músculo-esqueléticos e diabetes. E no item Tratamento, os tópicos que deveriam ser citados referiam-se à reeducação alimentar e prática de atividade física.

Para o participante obter um ponto em cada um dos itens, sua resposta deveria corresponder a, pelo menos, metade dos tópicos elencados no banco de respostas para cada item. Caso a resposta não alcançasse o critério de correspondência igual ou maior que a metade dos tópicos no item que estava sendo analisado, o participante não recebia nenhum ponto no referido item. Desta forma, a classificação era finalizada da seguinte maneira: (a) Conhecimento satisfatório, quando o participante obteve cinco ou quatro pontos; (b) Conhecimento regular, quando o participante obteve três ou dois pontos; e, (c) Conhecimento insuficiente, quando o participante obteve um ponto ou não obteve ponto em nenhum item.

(2) Conhecimento sobre as orientações nutricionais

Nesta categoria foram analisados relatos dos participantes acerca de: (a) alimentos sugeridos para aumento do consumo, (b) alimentos sugeridos para diminuição do consumo, (c) horários indicados para as refeições e (d) lista de substituição de alimentos.

Para o participante obter um ponto em cada um dos itens, a resposta deveria conter pelo menos o relato de uma orientação registrada no Protocolo de orientação nutricional para crianças fornecido pelo profissional de nutrição. Estas informações eram individualizadas e, portanto, foram analisadas considerando-se cada participante. Caso a resposta não correspondesse a, pelo menos, uma orientação contida no Protocolo, o participante não recebia nenhum ponto no referido item. Desta forma, a categorização foi finalizada da seguinte maneira: (a) Conhecimento satisfatório, quando o participante obteve quatro ou três pontos; (b) Conhecimento regular, quando o participante obteve dois pontos; e, (c) Conhecimento insuficiente, quando o participante obteve um ponto ou quando não obteve nenhum ponto.

Para a análise do relato de adesão dos participantes às regras nutricionais sob o efeito do TRV e do TA, fez-se o cálculo do Índice de Adesão à Dieta (IAD) em todas as etapas da pesquisa, a partir de adaptação da fórmula utilizada por Nobre (2010). IAD: número de itens corretamente ingeridos dividido pela soma do número de itens recomendados com o número de itens incorretamente ingeridos. O resultado era multiplicado por 100 .

Foram considerados como itens recomendados aqueles descritos no Protocolo de orientação nutricional para crianças de cada participante. Os itens incorretamente ingeridos foram aqueles adicionados pelos participantes e que não foram recomendados pelo profissional de nutrição ou aqueles que foram ingeridos de maneira não correspondente ao prescrito pelo profissional de nutrição, quer na qualidade e/ou na quantidade. Os itens corretamente ingeridos foram aqueles relatados pelos participantes que correspondiam aos indicados no Protocolo de orientação nutricional para crianças, tanto em qualidade quanto em quantidade.

Para a análise da fidedignidade do IAD, foi selecionada aleatoriamente uma amostra de cada refeição (desjejum, lanche da manhã, almoço, lanche da tarde, jantar e ceia) das duas crianças participantes, bem como de todos os registros das refeições realizadas em um período de 24 horas (obtidos por meio do Recordatório 24 horas). Para o cálculo do Índice de Fidedignidade (IF), dois estudantes de Psicologia, com histórico de treino em análise de categorias de comportamentos e que não conheciam os objetivos do estudo, participaram como juízes independentes. Obtevese uma média de $78 \%$ no cálculo do IF entre os juízes e a pesquisadora.

$\mathrm{Na}$ análise dos tipos de erros alimentares, os itens incorretamente ingeridos foram categorizados de acordo com as seis categorias de erros alimentares elaboradas por Soares e Costa (2011) acrescidas de mais duas categorias (Tipo 7 e Tipo 8). As categorias eram: Tipo 1 (Alimento recomendado, mas ingerido em quantidade superior a indicada no Protocolo); Tipo 2 (Alimento não recomendado ingerido durante a refeição); Tipo 3 (Alimento não recomendado ingerido em intervalo entre as refeições); Tipo 4 (Alimento recomendado, mas não ingerido durante a refeição); Tipo 5 (Alimento recomendado ingerido em intervalo entre as refeições); Tipo 6 (Alimento recomendado, mas ingerido em refeição diferente daquela em que foi recomendado); Tipo 7 (Alimento recomendado, mas ingerido em quantidade inferior a indicada no Protocolo); e Tipo 8 (Refeição não realizada).

Os resultados obtidos foram analisados de modo longitudinal, a partir do delineamento de sujeito como seu próprio controle, considerando-se a adesão da criança 
ao tratamento nutricional ao início do estudo (LB1), após a aplicação do Manual informativo sobre obesidade e alimentação saudável, após o treino em relato verbal (TRV), em LB2, após o treino em registros de automonitoração (TA) e em Follow-up.

\section{Resultados}

\section{Análise dos efeitos do uso de instruções}

Analisando-se o conhecimento sobre obesidade, observou-se que P1 apresentou resultado correspondente a conhecimento regular tanto antes quanto após a aplicação do Manual informativo sobre obesidade e alimentação saudável. No caso de P2, os resultados indicaram conhecimento insuficiente antes e após a aplicação do Manual. No que se refere ao conhecimento das regras nutricionais, P1 demonstrou conhecimento satisfatório antes e após a aplicação do Manual. Por sua vez, P2 inicialmente apresentou conhecimento insuficiente sobre as regras nutricionais e após a aplicação do Manual demonstrou ter conhecimento regular a este respeito.

Os resultados indicaram que P1 manteve a mesma classificação ao início e ao final do estudo em todos os itens analisados acerca de conhecimento sobre obesidade e regras nutricionais, sugerindo que o uso do Manual não foi suficiente para modificar o conhecimento deste participante acerca da doença e das regras nutricionais. Quanto a P2, observou-se melhora no conhecimento das regras nutricionais ao final do estudo.

Análise dos efeitos do treino de relato verbal e do treino de automonitoração

$\mathrm{Na}$ Figura 1 estão apresentados os percentuais correspondentes ao Índice de Adesão à Dieta (IAD) obtidos pelos participantes P1 e P2 em linha de base 1 (LB1), treino

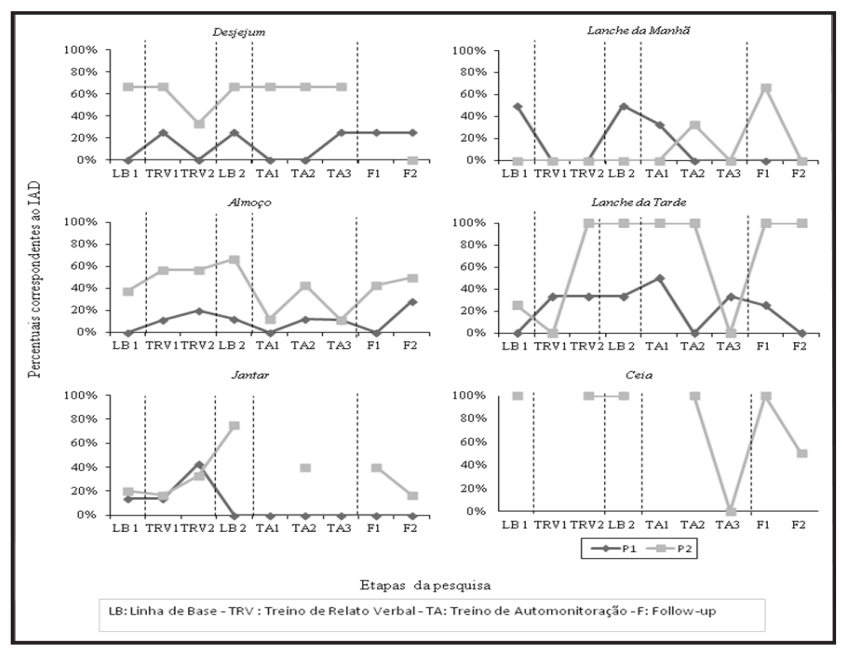

Figura 1. Índices de adesão à dieta obtidos pelos participantes P1 e P2 de acordo com as refeições indicadas no Protocolo de orientação nutricional e de acordo com a ordem do procedimento. de relato verbal (TRV), linha de base 2 (LB2), treino de automonitoração (TA) e em Follow-up (F), considerando-se cada uma das refeições.

Analisando-se a Figura 1, pode-se observar que os IADs de P2 foram mais elevados do que os obtidos por P1 em todas as etapas da pesquisa. Verifica-se que P1 obteve resultados iguais ou inferiores a $50 \%$ em todos os IADs, independentemente da refeição ou da etapa em que a coleta de dados foi realizada. O Jantar foi a refeição na qual P1 obteve IADs mais baixos. A ausência de registros referentes à Ceia justifica-se pelo fato deste participante não ter realizado esta refeição durante a coleta de dados.

P2 apresentou resultados superiores a 50\% na maioria dos IADs referentes ao Desjejum, Lanche da Tarde e Ceia. No Lanche da Manhã, os IADs de P2 ficaram abaixo de $50 \%$ em todo o período da pesquisa, apresentando elevação somente em Follow-up 1. No Almoço, os IADs de P2 ficaram próximos de $50 \%$ em TRV, ocorrendo decréscimo no período de TA, voltando a aumentar nas sessões de Follow-up. No Jantar, P2 obteve predominantemente IADs inferiores a 50\%.

Observa-se que a Ceia não foi regularmente realizada pelos participantes e que, considerando-se todos os IADs, somente P2 alcançou resultado igual a 100\% (com seis ocorrências no Lanche da Tarde e cinco na Ceia).

Quanto às médias percentuais dos IADs obtidos pelos participantes nas etapas da pesquisa, os resultados de P1 foram 11\% em linha de base 1 (LB1), 15\% em treino de relato verbal (TRV), 20\% em linha de base 2 (LB2), $9 \%$ em treino de automonitoração (TA) e 9\% em follow-up (F). Os resultados de $\mathrm{P} 2$ foram $42 \%$ em LB1, 39\% em TRV, 68\% em LB2, 36\% em TA e 51\% em F. A média entre os dois participantes em cada etapa foi a seguinte: $26,5 \%$ em LB1, $27 \%$ em TRV, 44\% em LB2, 22,5\% em TA e 30\% em F.

Comparando-se os dois participantes, observa-se que houve aumento de $39,77 \%$ entre os IADs obtidos nas LB1 e LB2, isto é, após treino em relato verbal, sugerindo mudança

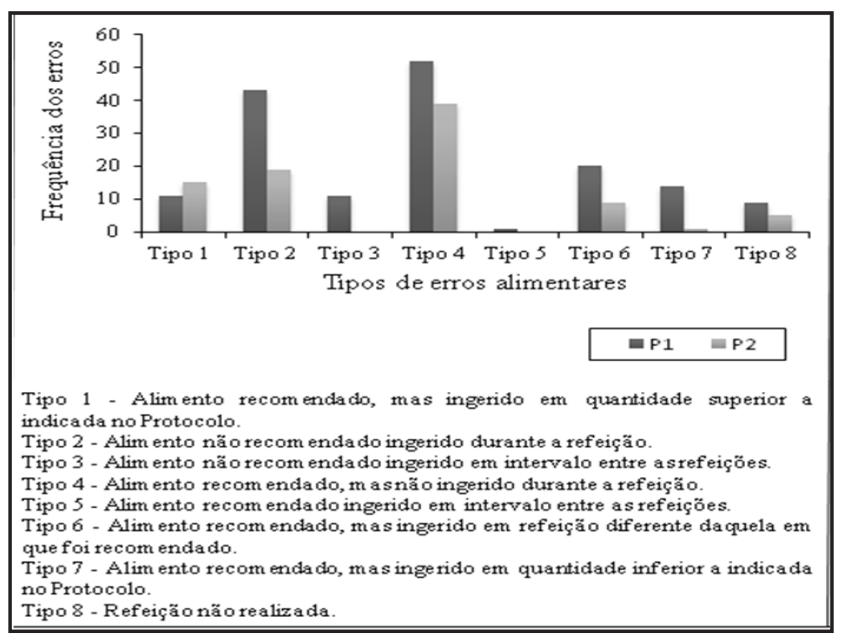

Figura 2. Comparação entre a frequência dos tipos de erros alimentares observados nos registros dos participantes P1 e P2. 
com significância clínica segundo Patterson, Chamberlain e Red (1982).

Análise dos erros alimentares apresentados pelos participantes

Na Figura 2 apresenta-se a comparação da frequência total de tipos de erro alimentar observados nos registros de P1 e de P2.

Observa-se na Figura 2 que os tipos de erro alimentar mais frequentes foram o Tipo 4 (Alimento recomendado, mas não ingerido durante a refeição) e o Tipo 2 (Alimento não recomendado ingerido durante a refeição). Comparando-se os dois participantes, observa-se que P1 apresentou maior frequência em todos os tipos de erro alimentar do que P2, com exceção do erro Tipo 1 (Alimento recomendado, mas ingerido em quantidade superior a indicada no Protocolo).

No que se refere aos erros menos frequentes, observa-se que, no caso de P1, foi o erro Tipo 5 (Alimento recomendado ingerido em intervalo entre as refeições). No caso de P2, os erros Tipo 3 (Alimento não recomendado ingerido em intervalo entre as refeições), o Tipo 5 (Alimento recomendado ingerido em intervalo entre as refeições) e o Tipo 7 (Alimento recomendado, mas ingerido em quantidade inferior a indicada no Protocolo) foram os menos frequentes.

A análise dos resultados relacionados aos erros alimentares observados em cada refeição indicou que o Almoço e o Jantar tiveram maior número de erros, independente do tipo, nos dois participantes. Em geral, prevaleceram os mesmos tipos de erros tanto para P1 quanto para P2 nas refeições, com exceção da Ceia.

Comparando-se os dois participantes, verificou-se que ao longo dos treinos o erro Tipo 1 (Alimento recomendado, mas ingerido em quantidade superior a indicada no Protocolo) diminuiu de frequência em ambos, o Tipo 2 (Alimento não recomendado ingerido durante a refeição) aumentou de frequência no caso de P1 e diminuiu no caso de P2 e o erro Tipo 4 (Alimento recomendado, mas não ingerido durante a refeição) aumentou de frequência no caso de P1.

\section{Discussão}

A combinação de variáveis manipuladas nesta pesquisa (instruções, treino em relato verbal e treino em automonitoração) mostrou-se favorável à ampliação do comportamento alimentar dos participantes tendo como referência o próprio sujeito ao longo do estudo, sugerindo a aplicabilidade do modelo construcional de Goldiamond (1974/2002). Essas variáveis correspondem ao que se denomina na literatura como controle por regras e treino de auto-observação; e, seu efetivo controle sobre o comportamento alvo tem sido investigado amplamente.

Desta forma, acredita-se que a manipulação dessas variáveis mostrou-se efetiva em ampliar o comportamento alimentar dos participantes, possibilitando melhora no seguimento das regras nutricionais. Tais resultados foram semelhantes aos observados nos estudos de Epstein et al. (1985), Gunnarsdottir et al. (2011) e Robertson et al. (2011) que enfatizaram a eficácia do uso de técnicas comportamentais para o tratamento do excesso de peso em crianças. Além disso, os resultados obtidos neste estudo concordam com a afirmativa de Fisberg (2004) referente à possibilidade de técnicas comportamentais auxiliarem na adesão ao tratamento a partir de mudanças no contexto familiar, como foi o caso de P2 que teve a cuidadora presente em todas as etapas do estudo e que relatou mudanças nos hábitos alimentares de outros membros da família.

Analisando-se os efeitos de instruções separadamente das demais variáveis, observou-se que, ao início do estudo, P1 tinha mais conhecimentos sobre obesidade e sobre as regras nutricionais necessárias ao tratamento ao ser comparado com P2; entretanto, seus IADs foram inferiores aos de P2 ao longo de todo o estudo. Considerando-se apenas a Linha de Base 1, tal resultado não está de acordo com a afirmação de Triches e Giugliani (2005) referente à associação entre maior conhecimento sobre regras nutricionais e maior frequência de comportamentos alimentares saudáveis. Porém, observou-se que P2, após ter sido submetido às instruções por meio do Manual, melhorou seu conhecimento sobre obesidade e sobre as regras nutricionais, assim como aumentou seus IADs. Desse modo, no caso de P2, confirmou-se tal associação.

Por sua vez, os treinos de relato verbal e de automonitoração mostraram efeitos semelhantes, não sendo observada maior eficácia de um em detrimento do outro ao longo do estudo. Entretanto, observou-se um aumento com significância clínica na média dos IADs dos dois participantes entre a LB1 e a LB2. Isto é, após o treino em relato verbal, houve melhora clínica no comportamento de seguir as regras nutricionais segundo o relato de ambos os participantes, de acordo com Patterson et al. (1982).

Quanto aos IADs (em sua maioria abaixo de 50\%) obtidos pelos participantes tanto em TRV quanto em TA, pode-se inferir que tais resultados confirmam a hipótese de que o contato tardio com as complicações da obesidade é um dos fatores que dificultam a adesão ao tratamento, de acordo com Malerbi (2000). Além disso, também confirmam a proposição de Albuquerque e Ferreira (2001) de que regras extensas, como as expostas no protocolo nutricional, são difíceis de serem seguidas.

Por outro lado, destaca-se que tanto TRV quanto TA permitiram a instalação de comportamento de autoobservação, o que pode ter favorecido relatos mais fidedignos do comportamento alimentar. Com isso, acredita-se que ambos os treinos podem ter sido úteis para o autoconhecimento e a auto-observação dos participantes, como observado nos estudos de Bentes (2011), Ferreira e Fernandes (2009), Hidaka (2007), Immer (2005), Nobre (2010) e Soares e Costa (2011). Como exemplos de tecnologia comportamental, TRV e TA podem auxiliar o profissional de nutrição na análise da adesão dos pacientes. Estudos futuros poderiam investigar o efeito da ordem de apresentação do treino para verificar se há diferença nos resultados obtidos caso a intervenção comece com automonitoração seguida de treino de relato verbal.

Os tipos de erros alimentares mais frequentes (Tipo 4 - alimento recomendado, mas não ingerido durante a refeição - e Tipo 2 - alimento não recomendado ingerido durante a refeição) encontrados nos dois participantes, independentemente da etapa do estudo, sugerem que o Protocolo de orientação nutricional provavelmente não estava de acordo com o repertório inicial dessas crianças, isto é, com o que era frequentemente oferecido e ingerido 
por estas. Valverde e Patin (2004) e Malerbi (2000) sugerem que há necessidade de se considerar o repertório inicial do paciente, isto é, os comportamentos adequados já instalados, e progressivamente aumentando as exigências de mudança. Esta proposição também está de acordo com o modelo construcional de Goldiamond (1974/2002). Por outro lado, verificou-se a importância de avaliar previamente o repertório de seguimento de regras dos participantes, ficando como sugestão para estudos posteriores.

Considerando as duas condições do estudo, observou-se que a presença da cuidadora revelou-se uma variável importante, uma vez que foram observados melhores resultados na adesão às recomendações nutricionais na Condição 2. Este resultado é semelhante aos obtidos nos estudos de Germann et al. (2007) e Kirschenbaum et al. (1984), e ainda, amplia discussões realizadas em pesquisas básicas sobre seguimento de regras com e sem monitoria, como, por exemplo, no estudo de Albuquerque et al. (2004), no qual verificou-se que o seguimento de regras mesmo monitorado é menos provável quando ocasiona perda de reforçadores, o que seria o caso de mudança no comportamento alimentar.

$\mathrm{O}$ fato de $\mathrm{P} 2$ ter demonstrado maior adesão ao tratamento quando comparado a P1, sugere que a presença do cuidador mostrou-se relevante nesta pesquisa, contrapondo os achados em pesquisas anteriores, as quais indicam que não havia diferença entre o tipo de intervenção, se somente com a criança ou se com a criança e o cuidador (Epstein, Paluch, Gordy, Saelens \& Ernst, 2000; Kingsley \& Shapiro, 1977). Por outro lado, coincide com a revisão sistemática realizada por Kitzmann e Beech (2006) que sugere que a participação do cuidador torna a intervenção bem-sucedida quando comparado com grupos controle, como também foi observado nos estudos de Golan, Weizman, Apter e Fainaru (1998) e Golan e Crow (2004). Desse modo, não está claro o papel da presença do cuidador, merecendo mais estudos nesta área, já que a literatura aponta resultados positivos tanto em intervenções realizadas somente com a criança, somente com o cuidador ou com ambos.

No presente trabalho, a ênfase estava no sujeito como seu próprio controle. Contudo, estudos posteriores poderiam trabalhar com uma amostra maior, além de parear variáveis sociodemográficas como sexo, idade e escolaridade dos participantes, idade dos cuidadores, estado clínico dos cuidadores e qualidade da relação entre cuidadores e crianças. Sugere-se ainda que pesquisas futuras avaliem os efeitos da presença do cuidador por meio de um delineamento A-B-A, no qual seria controlada a presença-ausência do cuidador com os mesmos participantes, em um delineamento de longo prazo, com o sujeito como seu próprio controle. Ou ainda, um delineamento com dois grupos, A-B-A e B-A-B.

Neste estudo, os dados foram coletados por meio do relato (oral em TRV e por escrito em TA) dos participantes. A literatura aponta que relatos referentes a comportamentos emitidos recentemente, como no período de 24 horas antecedentes à entrevista, são mais confiáveis (Smith, 1999). A confiabilidade do relato pode ser verificada também quando o mesmo é emitido sob o controle de perguntas relacionadas a comportamentos específicos (Malerbi, 2000). Nesta pesquisa, os resultados revelaram-se confiáveis, especialmente porque se observou predomínio de relatos de não seguimento das regras nutricionais, demonstrando que a postura terapêutica da pesquisadora, como audiência não punitiva, apresentando reforço social para o comportamento de relatar do participante, pode ser uma forma eficaz de ter acesso a comportamentos de adesão ao tratamento, o que também foi verificado nos estudos de Bentes (2011) e discutido por Malerbi (2000) e por Ferreira e Fernandes (2009).

Provavelmente, tanto TRV quanto TA alteraram o repertório inicial dos participantes relativo ao seguimento de regras, concordando, portanto, com a hipótese de que reforçar o relato de comportamentos de seguir regras aumentará a probabilidade de emissão destes comportamentos em ocasiões futuras, conforme sugere a literatura (Albuquerque, 2001; Baum, 2006; Blakely \& Schlinger, 1987; Schlinger \& Blakely, 1987; Skinner, 1969/1975).

Como contribuição desta pesquisa destaca-se a possibilidade de aplicabilidade dos instrumentos e do procedimento na prática de profissionais que trabalham diretamente com crianças com excesso de peso.

Uma das limitações deste estudo refere-se ao número reduzido de participantes. Isto ocorreu em parte pela dificuldade em conseguir mais crianças com diagnóstico de obesidade ou sobrepeso no ambulatório selecionado para este estudo, sugerindo que crianças com estas características podem não estar tendo acesso ao serviço prestado pelo HU. Outro motivo foi, provavelmente, a não aceitação dos cuidadores em participar da pesquisa devido à dificuldade de disponibilizar tempo para frequentar o hospital uma vez por semana, o que também foi enfatizado por McClaskey (2010), ao referir-se sobre as barreiras que profissionais da área da saúde enfrentam no tratamento da obesidade infantil. Ou ainda, por estes cuidadores não considerarem a obesidade/sobrepeso como uma doença que necessita de tratamento, uma vez que suas complicações geralmente são contatadas remotamente, como referido por Malerbi (2000). Tais hipóteses precisariam ser ainda investigadas.

Um diferencial deste trabalho foi utilizar o mesmo procedimento em crianças acompanhadas ou não pelo cuidador principal, uma vez que a maioria dos estudos opta por intervir somente com a criança, ou com ambos. Tratase de um estudo com limitações relativas ao número de participantes, contudo espera-se que outros trabalhos sejam realizados nesta linha de pesquisa a fim de confirmar ou refutar esses resultados.

\section{Referências}

Albuquerque, L. C. de. (2001). Definições de regras. In H. J. Guilhardi, M. B. B. P. Madi, P. P. Queiroz \& M. C. Acoz (Eds.), Sobre comportamento e cognição: expondo a variabilidade (pp. 132-140). Santo André: Arbytes.

Albuquerque, L. C. de, \& Ferreira, K. V. D. (2001). Efeitos de regras com diferentes extensões sobre o comportamento humano. Psicologia: Reflexão e Crítica, 14, 143-155.

Albuquerque, N. M. A. de, Paracampo, C. C. P., \& Albuquerque, L. C. de. (2004). Análise do papel de variáveis sociais e de consequências programadas no seguimento de instruções. Psicologia: Reflexão e Crítica, 17, 31-42. 
Associação Brasileira de Empresas de Pesquisa [ABEP]. (2010). Critério de classificação econômica Brasil. Retrieved from www.abep.org

Baum, W. M. (2006). Compreender o behaviorismo: ciência, comportamento e cultura. (M. T. A. Silva, M. A. Matos, G. Y. Tomanari, \& E. Z. Tourinho, Trads., 2 ed.). Porto Alegre: Artmed.

Bentes, T. F. (2011). Efeitos do uso de registros de automonitoração sobre o comportamento alimentar de crianças com sobrepeso (Trabalho de conclusão de curso). Universidade Federal do Pará, Belém.

Blakely, E., \& Schlinger, H. (1987). Rules: function-altering contingency-specifying stimuli. The Behavior Analyst, 10, 183-187.

Epstein, L. H., Paluch, R. A., Gordy, C. C., Saelens, B. E., \& Ernst, M. M. (2000). Problem solving in the treatment of childhood obesity. Journal of Consulting and Clinical Psychology, 68, 717-721.

Epstein, L. H., Wing, R., Woodall, K., Penner, B. C., Kress, M. J., \& Koeske, R. (1985). Effects of family-based behavioral treatment on obese 5-to-8-year-old children. Behavior Therapy, 16, 205-212.

Fagundes, A. J. F. M. (1982). Descrição, definição e registro de comportamento. São Paulo: Edicon.

Ferreira, E. A. P., \& Fernandes, A. L. (2009). Treino em autoobservação e adesão à dieta em adulto com diabetes tipo 2 . Psicologia: Teoria e Pesquisa, 25, 629-636.

Fisberg, M. (2004). Primeiras palavras: uma introdução ao problema do peso excessivo. In M. Fisberg (Ed.), Atualização em obesidade na infância e adolescência (pp. 1-9). São Paulo: Atheneu.

Fisher, J. O., Sinton, M. M., \& Birch, L. L. (2009). Early parental influence and risk for the emergence of disorder eating. In L. Smolak \& J. K. Thompson (Eds.), Body image, eating disorders, and obesity in youth: Assessment, prevention, and treatment ( $2^{\mathrm{a}}$ ed., pp. 17-33). Washington, DC, US: American Psychological Association.

German, J. N., Kirschenbaum, D. S., \& Rich, B. H. (2007). Child and parental self-monitoring as determinants of success in the treatment of morbid obesity in low-income minority children. Journal of Pediatric Psychology, 32, 111-121.

Golan, M., \& Crow, S. (2004). Targeting parents exclusively in the treatment of childhood obesity: long-term results. Obesity Research, 12, 357-361.

Golan, M., Weizman, A., Apter, A., \& Fainaru, M. (1998). Parents as the exclusive agents of change in the treatment of childhood obesity. The American Journal of Clinical Nutrition, 67, 1130-1135.

Goldiamond, I. (2002). Toward a constructional approach to social problems: Ethical and constitutional issues raised by applied behavior analysis. Behavior and Social Issues, 11, 108-197. (Trabalho original publicado em 1974)

Gunnarsdottir, T., Sigurdardottir, Z. G., Njardvik, U., Olafsdottir, A. S., \& Bjarnason, R. (2011). A randomized-controlled pilot study of Epstein's family-based behavioural treatment for childhood obesity in a clinical setting in Iceland. Nordic Psychology, 63, 6-19.
Hidaka, A. H. V. (2007). Tratamento comportamental de crianças com obesidade ou sobrepeso: estudo exploratório baseado no modelo construcional de I. Goldiamond (Unpublished master's thesis). Universidade de Brasília.

Immer, I. A. (2005). Efeitos do treino de correspondência sobre o seguimento de regras nutricionais por adultos com diabetes tipo 2 e obesidade (Trabalho de conclusão de curso). Universidade Federal do Pará, Belém.

Kingsley, R. G., \& Shapiro, J. (1977). A comparison of three behavioral programs for the control of obesity in children. Behavior Therapy, 8, 30-36.

Kirschenbaum, D. S., Harris, E. S., \& Tomarken, A. J. (1984). Effects of parental involvement in behavioral weight loss therapy for preadolescents. Behavior Therapy, 15, 485-500.

Kitzmann, K. M., \& Beech, B. M. (2006). Family-based interventions for pediatric obesity: methodological and conceptual challenges from family psychology. Journal of Family Psychology, 20, 175-189.

Kopelman, C. A., Roberts, L. M., \& Adab, P. (2007). Advertising of food to children: is brand logo recognition related to their food knowledge eating behaviours and food preferences? Journal of Public Health, 29, 358-367.

Laessle, R. G., Uhl, H., Lindeli, B., \& Müller, A. (2001). Parental influences on laboratory eating behavior in obese and nonobese children. International Journal of Obesity, 25(1), 60-62.

Lent, M., Hill, T. F., Dollahite, J. S., Wolfe, W. S., \& Dickin, K. L. (2012). Healthy children, healthy families: Parents making a difference! A curriculum integrating key nutrition, physical activity, and parenting practices to help prevent childhood obesity. Journal of Nutrition Education and Behavior, 44, 90-92.

Lindsay, A. C., Sussner, K. M., Kim, J., \& Gortmaker, S. L. (2006). The role of parents in preventing childhood obesity. The Future of Children, 16, 169-186.

Malerbi, F. E. K. (2000). Adesão ao tratamento. In R. R. Kerbauy (Ed.), Sobre o comportamento e cognição: Psicologia comportamental e cognitiva. Conceitos, pesquisa e aplicação, a ênfase no ensinar, na emoção e no questionamento clínico (pp.148-155). Santo André: ARBytes.

McClaskey, E. L. (2010). A childhood obesity program in federally qualified community health centers. Journal of Health Care for the Poor and Underserved, 21, 774-779.

Nobre, S. M. (2010). Efeitos do treino de automonitoração e do treino de relato verbal no estabelecimento e na manutenção de comportamentos de seguir regras nutricionais em adultos com obesidade (Unpublished master's thesis). Universidade Federal do Pará, Belém.

Patterson, G. R., Chamberlain, P., \& Red, J. B. (1982). A comparative evaluation of a parent-training program. Behavior Therapy, 13, 638-650.

Robertson, W., Thorogood, M., Inglis, N., Grainger, C., \& StewartBrown, S. (2011). Two-year follow-up of the 'Families for Health' programme for the treatment of childhood obesity. Child: Care, Health and Development, 38, 229-236.

Rossi, A., Moreira, E. A. M., \& Rauen, M. S. (2008). Determinantes do comportamento alimentar: Uma revisão com enfoque na família. Revista de Nutrição, 21, 739-748.

Schlinger, H., \& Blakely, E. (1987). Function-altering effects of contingency-specifying stimuli. The Behavior Analyst, 10, 41-45. 
Skinner, B. F. (1975). Contingências do reforço: Uma análise teórica. Os Pensadores (R. Moreno, Trad.). São Paulo: Abril Cultural. (Trabalho original publicado em 1969)

Smith, A. F. (1999). Concerning the suitability of recordkeeping for validating and generalizing about reports of health-related information. Review of General Psychology, 3(2), 133-150.

Soares, A. A. A., \& Costa, D. C. T. (2011). Adesão ao tratamento nutricional da obesidade infantil: análise comparativa dos relatos da diade cuidadora-criança (Trabalho de Conclusão de Curso). Universidade da Amazônia, Belém.
Triches, R. M., \& Giugliani, E. R. J. (2005). Obesidade, práticas alimentares e conhecimentos de nutrição em escolares. Revista de Saúde Pública, 39, 541-547.

Valverde, M. A., \& Patin, R. V. (2004). Aconselhamento dietético e mudança de comportamento. In M. Fisberg (Ed.), Atualização em obesidade na infância e adolescência (pp. 69-78). São Paulo: Atheneu. 